\title{
Artículos
}

\section{Portales de internet: concepto, tipología básica y desarrollo}

\author{
Por Juan Carlos García Gómez
}

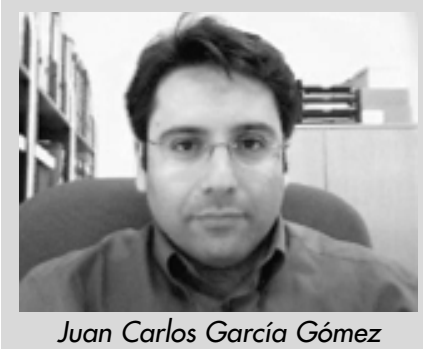

\begin{abstract}
Resumen: Se muestra la evolución experimentada por los primeros buscadores, índices y proveedores de servicios internet hacia su conversión en portales, los cuales se pueden clasificar en portales generales, verticales y corporativos. Se analizan algunas de sus características diferenciadoras y la razón de su presencia en internet, así como los principales elementos que favorecen su éxito, entre los que destaca la generación de contenidos, creación de comunidades virtuales, la potenciación de la fidelización del usuario y el desarrollo del
\end{abstract} comercio electrónico.

Descriptores: Portales web, Portales verticales, Portales corporativos, Comercio electrónico, Servicios de valor añadido en internet.

Title: Internet portals: concept, characteristics and development

Abstract: This paper shows the evolution of the first internet search engines, indexes and service providers towards their conversion into portals. These can be grouped into three classifications: general, vertical, and corporate. Some distinguishing characteristics of portals are analyzed as well as the reasons behind their existence in internet. Also discussed are the main elements which favour their success, including: generation of contents, creation of virtual communities, promotion of user loyalty and development of e-commerce.

Keywords: Web portals, Vertical portals, Corporate portals, E-commerce, Added value services in internet.

García Gómez, Juan Carlos. "Portales de internet: concepto, tipología básica y desarrollo". En: El profesional de la información, 2001, julio-agosto, v. $10, n .7-8, p p .4-13$

\section{Introducción}

Con bastante frecuencia se puede leer o escuchar, tanto en un contexto coloquial como científico, el término portal utilizado como sinónimo de lo que anteriormente o paralelamente se ha venido denominando "página web" o "sitio web". En un alto grado, este empleo inadecuado del término obedece a un conocimiento imperfecto de su significado, connotaciones y principales características, así como a que el uso de dicha palabra se ha extendido y popularizado rápidamente. Por ello trataremos de clarificar mediante este texto, en la medida de lo posible, el concepto de portal y describir sus tipologías y formas de implementación.

La manera en la que instituciones y empresas desarrollaban su presencia en la Red ha experimentado una evolución en los últimos años. Al principio de su popularización se tendía a aparecer en ella de cualquier manera, lo que hizo surgir infinidad de pequeñas páginas corporativas en las que se ofrecía una información básica sobre la empresa o institución en cuestión: los datos fundamentales de contacto, alguna información general y en pocos casos se aportaban datos más profundos.
Posteriormente este modelo de proyecto web dejó de resultar efectivo para sus responsables. La simple presencia se tornó insuficiente y varios de estos sitios fueron incorporando algunos servicios de valor añadido y mejorando los contenidos, mientras que otros todavía conservaron ese primer modelo de presencia mínima. Para los primeros, ahora no basta con "estar" sino que es preciso "hacer". Ya no se plantean estrategias pasivas hacia la clientela, sino que emplean métodos más agresivos, más activos, en dura competencia por la captación de usuarios y, sobre todo, por su fidelización respecto a su producto o institución. Este tipo de organización y de sitio es el que está más cercano a encajar en la descripción de portal que planteamos.

\section{Orígenes y evolución: de las homepages a los portales}

Desde que empezaron los primeros intentos de $A r$ panet a finales de los años 60 , aparecieron problemas relativos a la clasificación y estructuración de los recursos distribuidos a lo largo de la Red al no ser posible establecer un método apriorístico que sistematizara el acceso a dicha información. Desde esos inicios se hicieron necesarias herramientas o métodos para faci- 


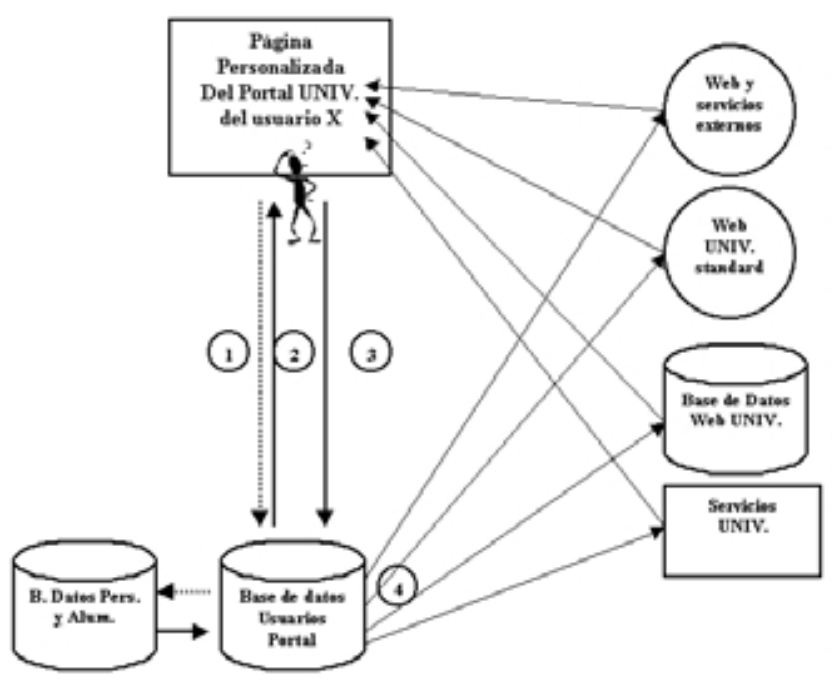

Figura 1

litar la navegación y la localización de las cada vez más numerosas fuentes disponibles.

Por ese motivo se desarrollaron sitios web cuya finalidad principal era organizar ese incipiente acerbo de conocimiento en línea. En 1994 surge Yahoo! con el objetivo de indizar las páginas existentes. Un equipo humano recopilaba información y la clasificaba dentro de su índice de categorías, cubriendo alrededor de 200.000 páginas (aproximadamente el 20\% del 1.000.000 de las existentes por entonces), ofreciendo la posibilidad de recuperar información a través de su índice de categorías y, accesoriamente, mediante un motor de búsqueda ${ }^{2}$.

http://www.yahoo.com

Con el paso del tiempo los sistemas de indización se hicieron más sofisticados: a veces incorporaron un robot para recoger información sobre las páginas que encontraban. Los robots son un tipo de programa cuya labor es rastrear continuamente la Red de forma automática, seguir los enlaces contenidos en los ficheros html y extraer información de algunas de sus partes tales como el título, texto alternativo de las imágenes, metaetiquetas, etc., siendo posteriormente almacenada en sus bases de datos de recursos.

De esta evolución surgieron dos sistemas de recuperación de información con significativas diferencias: de un lado los denominados "índices", en los que un grupo de personas elaboraba una enumeración de categorías en donde se clasificaban los contenidos ( $\mathrm{Ya}$ hoo!); de otro los llamados "buscadores", basados en un motor de búsqueda, como Altavista. Ambos tenían un mecanismo con el que poder lanzar consultas, pero en el caso de los primeros lo que destacaba en la página, tanto en diseño, presencia en pantalla, como en cuanto a potencia, era fundamentalmente la lista de clases ordenadas, más que el motor de búsqueda que incorporaban. En cambio, en el caso de los buscadores, el elemento destacado en la página era el formulario de consulta, desde el que se lanzaban las peticiones al motor, que trabajaba contra su base de datos de páginas web.

\section{http://www.altavista.com}

Posteriormente mejoró la calidad y capacidad de trabajo de los robots y, por lo tanto, el número de páginas que eran capaces de rastrear, pero pese a ello su número era siempre mucho menor que el total de las existentes, en crecimiento exponencial. Se estima que esta cifra se sitúa en la actualidad, según aproximaciones, en más de 1.000 millones (resultaría difícil aventurar cuántos millones más) de las que los robots de Altavista o HotBot, considerados de los más potentes, no consiguen indizar más de la tercera parte ${ }^{3}$. Se hizo necesario reconocer como imposible la labor de registrar todo el contenido de internet, cuyo volumen de páginas está tendiendo a sobrepasar ampliamente los límites de lo óptimamente gestionable. Ello fue una de las causas de la evolución de las páginas web de estos lugares. Al tiempo, evolucionaban las posibilidades técnicas y las necesidades de los usuarios, con lo que se fueron incorporando servicios de valor añadido que ahora es habitual encontrar en los portales (mensajes SMS, e-mail, espacio web, etc.).

http://www.hotbot.com

\section{«Ya no se plantean estrategias pasivas hacia la clientela, sino que emplean métodos más agresivos en dura competencia por la captación de usuarios, por su fidelización»}

Otra vía por la que se evolucionó hasta lo que ahora conocemos como portales, fue a través de las páginas de los grandes proveedores de servicios internet como AOL, Microsoft o Netscape, que estaban configuradas por defecto como páginas de inicio en sus navegadores. Muchos usuarios iniciaban a diario la navegación desde ellas al no haber modificado la configuración de sus ordenadores, con lo que recibían gran cantidad de visitas cada día y rentabilizaban de esta forma la publicidad albergada en ella (que habitualmente se paga por número de clicks recibidos). Estos sitios implantaron contenidos atractivos y servicios de valor añadido con los que se trataba de potenciar el tráfico recibido $\mathrm{y}$, como objetivo último, fidelizar al usuario.

\section{http://www.aol.com}

http://www.microsoft.com

http://www.netscape.com 


\section{Definición de portal}

A la hora de hacer una descripción del concepto es posible encontrar alguna mucho más exigente que otra al ofrecer el calificativo de portal para un sitio web. Se podría ofrecer una primera, bastante básica, diciendo que es un punto de entrada a internet donde se organizan sus contenidos, ayudando al usuario y concentrando servicios y productos ${ }^{4}$, de forma que le permitan realizar cuanto necesite hacer en la Red a diario, o al menos que pueda encontrar allí todo cuanto utiliza cotidianamente sin necesidad de salir de dicho sitio. El objetivo último, como ya hemos comentado, pretende ser su fidelización, es decir, conseguir que no lo usen de forma eventual sino que se habitúen a hacerlo a diario, lograr que vuelvan en repetidas ocasiones con expectativas de encontrar servicios que normalmente usan en internet, información interesante y que se establezca algún tipo de vínculo casi personal entre el usuario y el portal. Se persigue no sólo que los visitantes coloquen la página en su bookmark o lista de URLs favoritas, sino que sea por defecto la de inicio de su navegador, lo que garantizaría en el futuro un tráfico alto y constante. Esto ayudaría a asegurar su supervivencia por un lado por medio de ingresos derivados de la publicidad en forma de banners (pequeños anuncios incrustados en la pantalla), y por otro gracias a servicios adicionales como venta de productos o comercio electrónico.

1. Portal vs. comunidad virtual. Tanto un término como otro se está utilizando en muchos casos para definir lo mismo. Si bien es muy cercana y estrecha la relación entre ambos, consideramos que existe una forma sencilla de diferenciarlos. Podríamos decir que una comunidad virtual es un conjunto de individuos (personas), entre los que no se establece necesariamente un contacto físico, que poseen un conjunto de necesidades de información comunes, que tienen una afinidad más o menos clara según su profesión, aficiones, relación geográfica o lingüística, etc. Este grupo necesita un espacio virtual común en la Red en torno al que trabajar, reunirse virtualmente, recibir e intercambiar información, utilizar servicios de gestión o comunicación, etc. ${ }^{5}$, y que sería un portal orientado a dicho colectivo.

2. Características diferenciadoras de un portal frente a una página web. Profundizando en la definición de portal es preciso matizar un poco más esa primera acepción básica. Si nos limitáramos a afirmar solamente que es "un punto de acceso a internet" se aportaría muy poco, pues casi cualquier página personal podría serlo sin ser un portal propiamente hablando. Un portal es, en todos los casos, un sitio web (website), una página, pero no viceversa; de igual forma ni cualquier página ni cualquier sitio web serían un por- tal. Tanto página como sitio web son conceptos con una clara relación de sinonimia. Podríamos matizar ambos afirmando que el segundo es un conjunto de páginas estructuradas sobre la base de una organización de contenidos rigurosos y que éstas se componen de una o más páginas, pero sin que necesariamente esté implícita la idea de estructuración de información con criterios rigurosos.

Aunque hemos encontrado bastantes definiciones y descripciones del término portal que lo analizan convenientemente, consideramos interesante utilizar la lista de características fundamentales que sugiere David Morrison (técnico especialista de Lotus en el International Technical Support Organization Center en las oficinas centrales de la compañía en Cambridge) que pueden ayudar a reconocer un portal frente a otro tipo de páginas web. Para ello, el autor usa las iniciales del término ${ }^{6}$ :

-Personalización para usuarios finales.

—Organización del escritorio.

- Recursos informativos divididos y organizados.

- Trayectoria o seguimiento de las actividades de los usuarios (tracking).

-Acceso a bases de datos.

- Localización de gente o de cosas importantes.

El aspecto fundamental que subyace en estas características se encuentra relacionado de forma directa con la personalización. Mejorando esta adaptación hacia el usuario y consiguiendo así uno "a medida", se podrían optimizar aspectos cruciales como la dificultad de navegación y localización de información relevante de entre aquella que no lo es, o la problemática para la interacción del portal con las actividades cotidianas, especialmente en situaciones de alta movilidad del usuario.

Muchos de los sitios web existentes en la actualidad intentan ofrecer toda la información que poseen mediante páginas con una estructura y jerarquización estándar, idénticas para todos los públicos, creadas y organizadas de la manera que los diseñadores consideraron más adecuada. Aunque un sitio esté bien diseñado y estructurado, será muy difícil navegar por él si contiene varios miles de enlaces y ha de ser válido para distintos tipos de usuarios y de objetivos.

Un buen portal debería identificar a quien ya lo ha visitado y permitirle personalizar tanto la interface (la visualización de la pantalla o "escritorio") como los contenidos, de forma que aquellos datos que se muestren estuvieran relacionados al máximo con él: ya fuere la cotización de sus acciones, el tipo de cambio de una moneda determinada, un calendario con fechas re- 
levantes (el cumpleaños de su pareja, un examen, una reunión importante, etc.). Estos datos habrán de ser los adecuados a su perfil o rol dentro de una institución, adaptados a sus preferencias o necesidades, lo que producirá que el aspecto formal y la distribución de contenidos de un portal que utilizan dos usuarios podrá diferir por completo, aun tratándose en realidad del mismo para ambos?

Esta configuración debería ser automática a partir de los datos del visitante que el sistema ya posea y que, en el caso de portales verticales (temáticos), serán introducidos por el propio usuario en el momento de darse de alta. En los corporativos podrá aportarlos la propia organización a partir de sus bases de datos de personal.

\section{«Se hizo necesario reconocer como imposible la labor de re- gistrar todo el contenido de in- ternet, cuyo volumen de pági- nas está tendiendo a sobrepa- sar ampliamente los límites de lo óptimamente gestionable»}

Uno de los objetivos de un buen portal debería ser que se pareciera al máximo al escritorio del usuario, de forma que desde él pudiera recibir información de su interés, además de manejar las aplicaciones informáticas con las que trabajase a diario.

Es posible utilizar varios métodos para la configuración y personalización. Los fundamentales son el uso de cookies y el acceso identificado. El inconveniente de las primeras es que implican que el usuario utilice siempre el mismo ordenador y que, además, no sea compartido por otros, cosa incompatible con el uso de máquinas de acceso público. La otra dificultad estriba en que la misma persona puede acceder desde distintos lugares (oficina, casa, sala de ordenadores, un ordenador de uso temporal) dificultando que las cookies puedan recoger unívocamente su propia información.

Los gestores del portal podrían utilizar la información derivada de la identificación del usuario para dos objetivos básicos. Desde el punto de vista del servicio prestado, puede reconocerlo tanto a él como a sus preferencias y, a partir del seguimiento de sus actividades, anticipar lo que necesitará para tratar de adelantarse en lo posible a ello. Por otro lado, comercialmente se conseguiría que la personalización se extienda también a las campañas de marketing para ofrecerle los anuncios, productos y servicios que mejor se adapten a sus características (su target), hecho que multiplica la efectividad de las campañas de promoción.
3. Ejemplo de funcionamiento de un portal universitario. Para ver de un modo más gráfico cómo debería funcionar un verdadero portal, especialmente en lo que se refiere a sus características de personalización y flujo de datos, veamos el ejemplo de uno de naturaleza corporativa para una universidad. Un sistema web típico actual podría tener del orden de entre 100 a varios miles de páginas. Ello dificulta realizar una clasificación y distribución de contenidos que satisfaga plenamente a todos los miembros de la comunidad universitaria. Si a cada individuo se le diera la posibilidad de elegir la información y enlaces que habría de contener su página ideal de inicio de navegación, aparecerían cientos de combinaciones distintas.

Cada persona integrada en la universidad, ya sea por su rol de profesional o alumno, su puesto de destino, los estudios en curso o simplemente por sus preferencias, necesita acceder rápidamente desde su página a determinada información y partes del web. Estos contenidos no tienen por qué parecerse a los elegidos por otros miembros de la comunidad universitaria. Un alumno podrá desear tener, por ejemplo, en su página de inicio el calendario de sus exámenes, cursos en vigor relacionados con sus estudios o sus intereses, acceso directo a su correo electrónico y vínculos a diversos recursos de ocio. Un profesor (PDI) es posible que quiera tener en ese inicio acceso al listado de sus alumnos, los horarios de su facultad, enlaces a publicaciones científicas o alertas sobre las nuevas publicaciones y recursos de investigación. Algo similar ocurriría con el personal de administración y servicios (PAS), becarios de investigación, titulados, etc., dependiendo de su puesto de trabajo e intereses personales.

Se pide al usuario un login y una contraseña para su identificación inicial. A partir de estos datos, cruzándolos con las bases de datos de empleados (PAS y PDI) y las de alumnos, el sistema obtiene un perfil inicial gracias al cual se le muestra una primera página de inicio configurada de acuerdo con su rol en la institución. A continuación se pueden personalizar los contenidos, añadiendo y eliminando posibilidades y opciones de acuerdo con sus intereses personales, modificando así la configuración original.

De este modo lo que anteriormente era una página estática corporativa de la universidad pasa a basarse en cuatro elementos fundamentales:

- Las bases de datos de personal y alumnos: con su información, el sistema creará una primera distribución de sus contenidos para cada usuario.

-Una base de datos de usuarios: en ella se guardarán los datos de configuración, obtenidos del cruce con las bases de datos de personal y alumnos, así co- 
mo de las posteriores modificaciones introducidas por el mismo.

-Una base de datos de páginas web: gestiona la parte dinámica del web "clásico" o estándar, con la que se controlarán todas las páginas susceptibles de cambios constantes o con plazos de caducidad concretos (convocatorias, becas, cursos, etc.).

-El sistema de páginas previamente existente (web "clásico").

$\mathrm{Al}$ entrar (figura 1) el usuario se identifica (proceso 1) y la primera base de datos del portal comentada anteriormente busca su configuración, que será aquella que el sistema haya preparado a partir de la consulta contra la base de datos de personal y alumnos (proceso 2). A partir de ahí se le da la posibilidad de modificarla y personalizar su página, cambios que quedarán registrados en la base de datos de usuarios del portal (proceso 3). A continuación, se irán cargando los contenidos en su página principal (proceso 4) usando las siguientes fuentes:

-Páginas html previamente existentes en el web de la universidad (lo que denominamos "web clásico").

- Consulta a la base de datos de páginas a partir de los criterios por él definidos en la personalización.

- Recursos externos a esa universidad que el usuario ha incorporado al sistema durante la configuración.

- Del mismo modo se presentarán accesos directos a los servicios por él elegidos (correo electrónico, página personal, foros, etc.).

El modelo aquí comentado, orientado al portal de una universidad, podría a su vez adaptarse perfectamente a cualquier otro tipo de carácter corporativo haciendo mínimas modificaciones.

\section{Tipos de portales}

Debido a la proliferación del número de portales y de usuarios, así como al vertiginoso crecimiento de internet, se hace necesario clasificarlos en función del público al que van dirigidos, de la línea de contenidos que pretenden cubrir. Se podrían distribuir teniendo en cuenta muchos criterios y obtener clasificaciones amplias y profundas, pero se ha considerado oportuno realizar una ordenación lo más simple posible con el objeto de que sea más sencillo obtener una visión general de este fenómeno. Así, distinguiremos entre aquellos orientados a toda la población (generales), los dedicados a usuarios interesados en un tema concreto (especializados) y los destinados a las personas relacionadas con una empresa o institución (corporativos).
1. Generales (megaportales o portales horizontales). Están orientados a todo tipo de público y ofrecen contenidos de carácter muy amplio, siendo su pretensión cubrir las temáticas más demandadas. Suelen incorporar servicios de valor añadido tendentes a la fidelización en torno a comunidades virtuales, tales como espacio web gratuito, información de diverso tipo, personalización de la información, chat, e-mail gratuito, mensajes a teléfonos móviles, software de libre distribución, grupos de discusión, comercio electrónico o buscador.

\section{«Un buen portal debería identifi- car a quien ya lo ha visitado y permitirle personalizar tanto la interface como los contenidos, de forma que aquellos datos que se muestren estuvieran re- lacionados al máximo con él»}

Este modelo tiende a resultar obsoleto. Se estima que sólo un número muy reducido puede ser capaz de ofrecer el adecuado nivel de servicios y contenidos a un público tan diverso y, al tiempo, ser viable organizacional y económicamente. Ello hace que resulte insuficiente para los usuarios más expertos ${ }^{8}$ y profesionales que exigen mayor especialización y profundidad, tanto en los servicios como en los contenidos, quedando entonces orientados fundamentalmente hacia los más inexpertos, habitualmente menos exigentes. Por tanto, la tendencia que se apunta tiende hacia la especialización geográfica, temática o corporativa.

2. Especializados. Cada vez hay más usuarios a los que, ya sea por su grado de experiencia o por sus necesidades profesionales, los comentados en el punto anterior no satisfacen convenientemente sus necesidades porque sus contenidos son excesivamente globales $\mathrm{y}$, por ende, demasiado superficiales e insuficientes para lo que sus características personales o profesionales demandan. Ello plantea una situación en la que existe una coyuntura favorable para aplicar el modelo de portal a aspectos más específicos. Se intentará cubrir, por ejemplo, un área geográfica determinada (la región de Murcia), un tema concreto (la educación familiar) o las necesidades de las personas relacionadas con una corporación específica (empleados, directivos, clientes, proveedores, etc.). Esta cobertura la ofrecen los portales verticales y corporativos.

http://www.tubitacora.com

http://www.familyeducation.com/

2.a. Corporativos. Es una intranet que provee información de la empresa a los empleados, así como de acceso a una selección de webs públicos y de mercado 
vertical (proveedores, vendedores, etc.). Incluye un motor de búsqueda para documentos internos y la posibilidad de personalización para diferentes grupos de usuarios y particulares. Sería el equivalente interno a los de carácter general ${ }^{9}$.

Tienden a ser una prolongación natural de las intranets corporativas, en las que se ha cuidado la organización de la información y la navegación; donde se permite, y sobre todo se potencia, el acceso a datos de la propia institución, la edición de material de trabajo propio, el contacto con clientes y proveedores, etc. En ellos se distingue la parte intramuros, o del cortafuegos hacia adentro, y la externa dependiendo de que el destinatario de esa información sea miembro de la institución o bien un elemento ajeno a ésta ${ }^{10}$.

2.b. Verticales —vertical portals (vortals) —. Es un sitio web que provee información y servicios a un sector o industria en particular. Es el equivalente industrial específico de los generales ${ }^{11}$, pero en este caso, además de ofrecer los típicos servicios de valor añadido característicos de éstos, la cobertura de sus contenidos se centra en un tema o área concreta.

Han de saber captar parte de los usuarios que los generales ya no son capaces de atender. Su potencial para ello estriba en la posibilidad de profundización en los contenidos que ofrecen y en su oferta de servicios personalizados. A su vez es posible clasificarlos dentro de otros dos grupos fundamentales, atendiendo a su tipo de especialización:

-Geográficos: centrados específicamente en una zona o área concreta. Por ejemplo: Toronto.com (especializado en esta ciudad canadiense).

http://www.toronto.com

- Temáticos: atienden a una línea temática. Un ejemplo claro sería el de Infonomía, especializado en la gestión de información y documentación empresarial.

http://www.infonomia.com

\section{Factores clave del éxito de un portal}

Los motivos fundamentales de un portal para aparecer en internet están íntimamente ligados con los objetivos de sus auspiciadores, lo que diferencia dos grandes grupos: los que no tienen ánimo de lucro y aquellos con carácter claramente comercial. En ambos casos el modo de gestión es similar en un aspecto: todos pretenden perpetuarse en la medida de lo posible. Para ello se hace necesario en un caso generar ingresos y en el otro atraer y mantener fieles a sus usuarios y subsistir económicamente, ya sea a partir de dinero público, privado, autogenerado o de los tres tipos.
Incluso las instituciones más típicamente no lucrativas deberían hacer una gestión eficiente de recursos que signifique un compromiso entre los objetivos institucionales y la viabilidad económica del proyecto. Por ello, puesto que ambos modelos pretenden subsistir, apuntamos una serie de ideas de gestión útiles a la hora de plantear un proyecto serio ya sea destinado a intentar generar beneficios o bien con la idea de cubrir los costes de desarrollo y mantenimiento inherentes al mismo.

Existen varios factores favorecedores de la generación de negocio, siendo los fundamentales el tráfico generado o visitas recibidas y el comercio electrónico creado, estando ambos conceptos directamente relacionados entre $\mathbf{s i ́}^{12}$.

Consideramos que el aspecto más importante para la atracción de tráfico al portal es la producción de materiales interesantes para el visitante. Conseguir gran número de visitas puede suponer, además de prestigio y difusión de los contenidos, ganancias directas por publicidad por medio de los ya citados banners. Con éstos los ingresos se suelen cifrar según el número de veces que se muestran o se hace click en ellos siendo, por tanto, los beneficios recibidos por este capítulo directamente proporcionales al publico que ha pasado por él ${ }^{13}$.

\section{«Incluso las instituciones más típicamente no lucrativas debe- rían hacer una gestión eficiente de recursos, que signifique un compromiso entre los objetivos institucionales y la viabilidad económica del proyecto»}

En el caso de portales comerciales este dinero se puede aumentar considerablemente si se consigue fomentar el comercio electrónico. La posibilidad de ampliar el mercado es para el 60\% de las empresas españolas el principal estímulo para el crecimiento de este nuevo método de negocio. Como barreras a su desarrollo se presentan aspectos como la poca familiaridad con la informática e internet, la falta de adecuación del producto al canal o la inexistencia de cultura empresarial ${ }^{14}$.

La realidad es que hasta ahora no ha terminado de explotar el comercio electrónico en los portales tal y como se preveía. Entre los motivos que se plantean para justificar este escaso crecimiento se puede citar la desconfianza existente por parte de muchos usuarios a la hora de usar tarjetas de crédito, si bien el fraude en la Red es porcentualmente la décima parte del producido en el mercado tradicional ${ }^{15}$. Éste y otros obstáculos deben ser superados por aquellos portales que quie- 
ran participar del negocio que se prevé en el futuro. Forrester Research estima que el 5\% de las ventas mundiales en el 2003 se realizarán a través del www.

\section{http://www.forrester.com}

Un modelo de portal de éxito lleva consigo la realización de acuerdos a la hora de abordar proyectos ambiciosos, de forma que se implique a distintas organizaciones y empresas especializadas en aspectos como diseño, marketing, seguridad en el comercio y, sobre todo, generación de contenidos atractivos. Todas deben aportar lo mejor de sí mismas para conseguir un servicio de calidad.

Otra posibilidad de difusión es aplicar formas de marketing distintas a la típica publicidad de banners (cuya efectividad se viene estimando en un $2 \%$ de clicks de los mostrados) como pueden ser los patrocinios $^{16}$. Con ellos se muestra un producto o marca en un lugar directamente relacionado con su público potencial, lo que conlleva resultados mucho mejores, de hasta el 7\% en algunos casos, al asociarse de un modo más natural la imagen y el nombre comercial del anunciante con la temática de los contenidos del portal. Un ejemplo de esto podría ser un artículo relacionado con la gestión documental que patrocinara determinadas secciones de un portal para profesionales de la información y documentación.

\section{«La realidad es que hasta ahora no ha terminado de explotar el comercio electrónico en los portales tal y como se preveía»}

También es posible generar ingresos a través de la venta de productos, que puede hacerse directamente si es el propio portal el que dispone tanto de la mercancía a vender como de la infraestructura adecuada y monta por sí mismo un servicio de tienda virtual. Otra posibilidad, en el caso de que los propietarios del portal no dispongan por sí mismos del material, es la de recurrir a los programas de afiliados mediante los que el portal hace de intermediario entre el usuario y otras empresas especializadas en la venta a través de la Red. Esas compañías ofrecen a los visitantes productos relacionados con la temática del portal, lo que les genera ingresos de comisiones por la venta realizada. Un ejemplo sería la posibilidad de compra de cd-roms regrabables u otros dispositivos de almacenamiento de información desde la sección de "conservación de materiales" de un portal para profesionales de la información.

En definitiva se debe tratar de aprovechar las posibilidades de internet, entorno muy distinto de los sistemas, procesos y modelos tradicionales de gestión, para tratar de integrar contenidos y negocio de la for- ma más natural posible. De esta manera nos encontramos con la posibilidad de que alguien realice una consulta desde el buscador del portal y, como resultado, localice un producto interesante que pueda comprar con un simple clic. Así se facilita la compra al cliente que en un principio no pensaba adquirir nada, pero al que le ofrecemos algo que anteriormente no conocía y que ahora se le muestra a su alcance de modo sencillo y útil ${ }^{17}$. Por ejemplo, tras buscar información sobre diseño eficiente de páginas web localiza un portal sobre gestión de información y documentación y halla un texto relativo a lo que buscaba, un resumen o una reseña de un libro que trata con profundidad el tema. $\mathrm{Pa}$ ra su compra sólo habría que pinchar en el icono correspondiente que se muestra en esa misma página.

Según los resultados de una encuesta realizada a un grupo de expertos ${ }^{18}$, algunos factores clave de éxito de los portales que han de asegurar la generación de negocio pueden ser: capacidad financiera, notoriedad y marca, alianzas estratégicas, promoción online y offline, nivel de los contenidos, servicios de valor añadido, sistema de búsqueda, penetración en el mercado (visitas recibidas), sentimiento de comunidad y orientación a la localidad.

Las reglas de funcionamiento de internet no actúan del mismo modo que en el entorno tradicional. Respecto a los sectores que triunfan en el comercio electrónico, los que tienen una mayor presencia de contenidos (automoción) no son siempre los que generan mayor negocio (medios). Del mismo modo, no son las grandes empresas las que crean comercio, sino las pequeñas, con un $44 \%$. El factor clave es la eliminación de algunos de los canales de venta clásicos como barrera de introducción, así como a su mayor agilidad y capacidad de adaptación ${ }^{18}$. Es importante considerar estos hechos cuando se plantee un proyecto de portal en internet, independientemente de la finalidad inherente a dicha iniciativa.

En lo que ha de incidir de forma clara y decidida todo tipo de portal, ya sea o no comercial, es en los contenidos y en las ideas. En ocasiones se dedican importantes esfuerzos al logro de un diseño atractivo y espectacular tras el que hay mínimos contenidos e innovaciones. Debe existir una actualización con la adecuada frecuencia para generar en el usuario expectativas de desarrollo constante, lo que favorecerá visitas frecuentes.

Funcionará si se utilizan recursos para la generación de ideas de calidad que diferencien ese producto del resto, con las que se consiga gran resultado con poco esfuerzo ${ }^{20}$. Se trataría de encontrar soluciones viables a cuestiones como ¿es trasladable un modelo concreto de la vida "real" a la virtual?, ¿puede haber algo 
que tenga cabida en internet pero no en el mundo real?, o ¿hay en la Red suficientes usuarios potenciales para dicho portal?

\section{Desarrollo sostenible de portales}

En lo referente a su creación, ya sea de naturaleza vertical, corporativa o especialmente de carácter general, no se exige el mismo esfuerzo que crear una pequeña página personal. Se requerirá un equipo de trabajo amplio e interdisciplinar, una planificación adecuada, marketing y una suficiente inversión de capital, en ocasiones bastante considerable.

a. Equipo humano. Será necesario reunir un grupo todo lo amplio y cualificado que sea posible, que integre principalmente personal informático, profesionales de la información y documentación así como a especialistas en marketing y diseño.

b. Planificación. Sin una adecuada y cuidadosa organización será difícil que un portal consiga éxito y perdurabilidad en la Red. Es preciso tener en cuenta aspectos muy importantes como: cuál es el público objetivo, cuáles son sus necesidades e intereses y de qué modo pueden quedar satisfechos. Las tareas de diseño y planificación de un buen portal deberían llevar más del $25 \%$ del total del tiempo de desarrollo ${ }^{21}$.

Resulta idóneo abordar la construcción de un portal por fases, empezando por un proyecto piloto. Esto servirá como aprendizaje en tareas de desarrollo, posibilitará evaluar su dimensión real, detectar posibles errores y hacer un seguimiento del éxito obtenido. Se debería aplicar el conocido modelo de Vilfredo Pareto de $80 / 20$ y tratar de potenciar al máximo el $20 \%$ de contenidos o servicios que reportan el $80 \%$ de las visitas o resultados.

c. Herramientas. El rápido crecimiento del número de portales ha propiciado la aparición de software que ayuda a su construcción para ser diseñados y configurados a medida ${ }^{22}$, habitualmente adecuados en cuanto a precio y prestaciones para instituciones de tamaño medio-grande.

Son cada vez más abundantes las herramientas con las que se pueden resolver diversos aspectos de la gestión del comercio electrónico. Tal es el caso de la solución para la realización de facturas en línea que proporciona la aplicación Netscape BillerXpert ${ }^{23}$, que es una más de las orientadas al desarrollo del comercio electrónico a través de portales. Las tendencias apuntan a que en el futuro se reduzca drásticamente el número de los denominados portales generales tales como los que ofrece la plataforma iPlanet, fruto de la fusión de Netscape y AOL.

http://www.iplanet.com
Una tendencia en auge para la gestión de portales utiliza html dinámico asociado a bases de datos, facilitando la gestión y actualización de un alto número de páginas. Su funcionamiento se basa en la inclusión en ellas de código de determinados lenguajes de programación que se ejecutan en el servidor, donde son interpretados, obteniéndose como resultado una página html que aparece en el cliente web del usuario ${ }^{24}$.

d. Costes. La construcción de un portal coherente implica el desembolso de una cuantía de dinero considerable. Algunas estimaciones sitúan el gasto de puesta en marcha de un portal corporativo en torno a 30 ó 40 MPTA, pudiendo elevarse hasta un total de cerca de 250 (1,5 Ma). Estas cantidades son independientes del número de usuarios reales, pudiéndose situar en torno a 700 el mínimo necesario para rentabilizarlo ${ }^{25}$.

\section{«Aunque interesantes y necesa- rias, no resulta suficiente para un portal ofrecer recopilaciones de enlaces que apuntan a recur- sos dispersos por la Red»}

Sin embargo si se pretende realizar un proyecto de pequeña envergadura, partiendo de una página web que posea cierto valor añadido en cuanto a contenidos, pero carente de los servicios típicos de un portal, existen soluciones gratuitas y sencillas como las que ofrece Tuportal.com que permite la incorporación de determinadas aplicaciones como correo electrónico, chat, foros, bases de datos, etc., en unos pocos minutos y con un aspecto final totalmente transparente para el usuario, de forma que son llamados desde la página de usuario, manteniendo en todo momento el diseño creado por el mismo.

\section{http://www.tuportal.com}

e. Desarrollo de contenidos. Aunque interesantes y necesarias, no resulta suficiente para un portal ofrecer recopilaciones de enlaces que apunten a recursos dispersos por la Red. Se hace necesario que el portal genere materiales propios y no se limite a copiar o enlazar lo que ya existe en otros lugares. La clave está en la calidad y profundidad de contenidos. Ello implicará acciones como realizar un seguimiento informativo de determinados temas de actualidad, elaborar informes, ofrecer opinión a través de firmas invitadas o secciones claramente dedicadas a ello. En determinados casos será preciso ofertar productos de ocio como concursos, humor, juegos, etc. En definitiva, aspectos que permitan diferenciar el producto propio del resto y que el visitante abandone el portal con el deseo de volver en poco tiempo. 
Al plantearse los contenidos a incluir y el tratamiento que se le va a dar, evidentemente hay que tener en cuenta al usuario: qué desea y que podría desear y cuál sería la mejor manera de satisfacer dichas necesidades.

Por ello se tiende a potenciar su participación, que exprese su acuerdo o desacuerdo con un artículo determinado, que aporte reseñas de libros sobre el tema del portal, que intervenga en los foros de discusión y en la creación de encuestas o que aporte sus colaboraciones. Con ello se consigue que se sienta parte integrante, que todos se enriquezcan con los puntos de vista aportados, etc. El mejor conocimiento de sus gustos, necesidades y forma de pensar ha de servir como retroalimentación y base para la evaluación de lo acertado de la línea de contenidos que se esté siguiendo.

f. Marketing. Todos los esfuerzos podrían resultar baldíos si no se llevase a cabo algún tipo de actividad de marketing para promocionarlo y tratar de conseguir un elevado número de visitas.

Una vía de promoción consiste en las campañas de intercambio de banners. A cambio de insertar uno o más ajenos en el portal, se visualizará el banner propio en otros lugares de internet. Habitualmente se sigue una proporción de 2 a 1 . Por cada dos banners externos que son mostrados se visualizará una vez el propio en otro sitio. En los centros de intercambio se optimizan al máximo dichas campañas, procurando que los banners se muestren en los lugares con un público objetivo lo más parecido posible al usuario real del portal en cuestión. Las empresas que durante el año 1999 invirtieron en marketing en internet dedicaron el 59\% del dinero a los banners, mientras que fue un $28 \%$ el dedicado a los patrocinios ${ }^{26}$.

Otra importante forma de acceso por parte de los usuarios es a través de las consultas en buscadores. Como consecuencia se debe afinar al máximo en la elección del texto de las etiquetas html de título y las metaetiquetas de descripción de contenido: Author, Description y Keywords, que fundamentalmente significan un peso importante en la descripción que del portal recogerán los robots de los buscadores.

Una manera alternativa de publicidad consiste en lo que se denomina "marketing de guerrilla" o uso de métodos no convencionales de promoción ${ }^{27}$, en los que predomina más la imaginación y agresividad que la inversión económica. Dentro de este marco se sitúa el denominado "marketing viral", hacer que sean los propios usuarios quienes difundan el portal entre otros interesados en su temática mediante elementos del tipo "envíaselo a un amigo" o "recomienda esta página". Este procedimiento viene a ser el equivalente en la Red del clásico "boca a boca". También los patroci- nios, anteriormente comentados, serían una importante forma de promoción.

Otra vía de difusión consiste en los programas de afiliados, mediante los que una empresa especializada en comercio electrónico se asocia con diversos portales que no se basan en este concepto y que podrán ofrecer desde sus páginas la posibilidad de venta online de artículos relacionados de algún modo con su espectro temático. El acceso se produce desde el portal, aunque en realidad las ventas se llevan a cabo con la empresa de venta online, obteniendo el portal desde el que se ha realizado la venta una comisión a cambio. A todos estos métodos se deberían añadir los tradicionales sistemas de publicidad y marketing "offline".

\section{«Las tendencias de desarrollo de los portales apuntan a que en el futuro se reduzca drástica- mente el número de los denomi- nados generales»}

g. Fomento de comunidades virtuales. Un portal en torno al que no exista una comunidad virtual expresa o tácita difícilmente podrá subsistir o tener alguna razón de ser pues, además de ser ésta la que le da sentido, también será la que lo enriquezca con sus aportaciones, comentarios, definiendo con su cifra de visitas los apartados más o menos potentes. En el caso de que el portal incluya elementos relacionados con la generación de negocio, serán éstos los que intervengan en los procesos de comercio electrónico y publicidad incorporada.

Obviamente, para que exista una comunidad virtual importante alrededor los contenidos tienen que ser lo suficientemente acertados como para interesar a ese grupo de personas y además han de tener conocimiento de algún modo de la existencia del portal y de la importancia de éste respecto a la satisfacción de sus intereses personales.

\section{Conclusiones}

Las tendencias de desarrollo de los portales apuntan a que en el futuro se reduzca drásticamente el número de los denominados generales. Muchos habrán de desaparecer por ser poco competitivos, o bien buscar su espacio idóneo convirtiéndose en verticales, para los que se vislumbran mejores expectativas de éxito y encontrar así una cuota de mercado suficiente para sostenerlo.

Plantear un modelo de portal con éxito implica concentrar esfuerzos en la generación de tráfico y comercio electrónico, para lo que se hace necesario potenciar los contenidos y la incorporación de servicios de valor añadido en general. 
Internet desarbola algunos de los tópicos en cuanto a la gestión y modelos de éxito. No siempre triunfan los métodos tradicionales, con lo que la imaginación y la capacidad de adaptación son la clave. Emprender la labor de construcción de un portal es un asunto serio y puede implicar un gasto considerable, si bien se pueden encontrar soluciones económicas para proyectos más modestos.

Para su adecuada gestión no basta con una buena labor respecto a diseño y contenidos, sino que será necesario hacerlos llegar a unos usuarios potenciales. Esto implica la adopción de técnicas de difusión y marketing que lo acerquen al público idóneo.

\section{Notas y referencias bibliográficas}

1. Podemos encontrar esta idea, así como la base del esquema de clasificación de portales incluido en este texto en: García, Juan Carlos; Gómez, José Antonio. "Qué son y de dónde vienen los portales de internet: comparativa de algunos portales de ocio". En: Educación y biblioteca (pendiente de publicación).

2. Flores, Jesús. "Los motores de búsqueda en la gestión de información de las empresas". En: La estrella digital, 1999, 28 de septiembre. Consultado en: 24-04-00.

http://www.estrelladigital.es/ciberestrella/secciones/saber/saber5.htm

3. García Alonso, Juan. "El futuro de la búsqueda de información en internet”. En: Internet' 99. Consultado en: 24-04-00.

http://www.aui.es/biblio/libros/mi99/16futuro_busqueda.htm

4. Arnedo, Txema. "De portales a plazas; presente y futuro de los portales en internet". En: Internet' 99. Consultado en: 06-04-00.

http://www.aui.es/biblio/libros/mi99/3portales.htm

5. Para profundizar en estos aspectos podemos consultar: Sánchez, Vanessa; Saorín, Tomás. "Las comunidades virtuales y los portales como escenarios de gestión documental y difusión de información”. En: Anales de documentación, 2001, n. 4, 2001, pp. 215-227.

6. Morrison, David. Building successful portals. En: Group computing magazine, 2000, mayo-junio. Consultado en: 24-05-01.

http://archives.groupcomputing.com/index.cfm?fuseaction=viewarticle \&C ontentID $=110$

7. Boettcher, Judith; Strauss, Howard. "What is a portal, anyway?". En: Techtalk transcript. Consultado en: 01-08-00.

http://www.cren.net/know/techtalk/trans/portals_1.html

8. Cornella, Alfons. "Portal el último... Mensaje 423". Extra-Net. Consultado en: 24-05-01.

http://www.infonomia.com/extranet/index.asp? idm $=1 \&$ idrev $=1 \&$ num $=423$

9. Definición del concepto "corporate portal" de la TechEncyclopedia, disponible en:

http://www.techweb.com/encyclopedia/

10. Fitzloff, Emily. "Portal patrol. Corporate portals help early users control data deluge". En: Info world electric, 1999, 17 de mayo. Consultado en: 26-04-00.

http://archive.infoworld.com/cgi-bin/displayStory.pl?/features/990517portal.htm

11. Definición del concepto "vertical portal” extraída de la TechEncyclopedia, disponible en:

http://www.techweb.com/encyclopedial
12. Esta idea, así como muchas de las citadas en este apartado aparecen reflejadas en: Cornella, Alfons. Infonomía.com. La empresa es información, Deusto, 2000.

13. Algunas de las reflexiones de este apartado fueron comentadas en el curso de promoción educativa de la Universidad de Murcia: "Los portales de internet".

http://www.um.es/gtiweb/portales/

14. Cabo, Julián de. "El comercio electrónico en España: posicionamiento de las empresas españolas y previsiones de futuro". En: Internet' 99.

Consultado en: 24-04-00.

http://www.aui.es/biblio/libros/mi99/25comercio_electronico.htm

15. Bordner, Doug. "Web portals: the real deal". En: Information week, 1999, 8 de febrero. Consultado en: 26-04-00.

http://www.its.inmar-inc.com/wp/InmarWebportals.htm

16. Podemos ver interesantes ejemplos de diversas formas de patrocinio y soluciones publicitarias aplicables a los portales en: Jané, Xavier. "Patrocinio y acciones especiales en internet". En: Internet' 99. Consultado en: 24-05-00.

http://www.aui.es/biblio/libros/mi99/13patrocinio.htm

17. Cornella, Alfons. "Vender a quién no había pensado en comprar. Mensaje 365". Extra-Net. Consultado en: 24-05-01.

http://www.infonomia.com/extranet/index.asp? idm $=1 \&$ idrev $=1 \&$ num $=365$

También en: El profesional de la información 1999, v. 8, n. 7-8, jul.-ago., p. 45 .

18. Resultados de una encuesta sobre portales realizada a un grupo de expertos por David Boronat, cuyo resumen de resultados se puede consultar en:

http://www.ogilvyinteractive.es/portales/resumen.htm

19. Cabo, Julián de, op. cit.

20. Cornella, Alfons. "El valor está en las ideas. Mensaje 438". ExtraNet. Consultado en: 24-05-01.

http: $/ /$ www.infonomia.com/extranet $/$ index.asp? $i d m=1 \& i d r e v=1 \&$ num $=438$

21. Vortal tutorial: lesson 2: developing content and design. Consultado en: 01-08-00.

http://www.stylusinc.com/portal/tutorial/lesson2.htm

22. Hibbard, Justin. Portals proliferate-new tools integrate personal interests. TechWeb. Consultado en: 26-04-00.

http://www.techweb.com/se/directlink.cgi?IWK19990125S0043

23. Existe información detallada dentro del web de Netscape, en las direcciones:

Comunicado de prensa:

http://home.netscape.com/es/newsref/pr/newsrelease697.html

Página de preguntas más frecuentes y artículos técnicos sobre dicha aplicación:

http://help.es.netscape.com/faqs/billerxpertlx.html

24. Pastor, Juan Antonio. Construcción de portales. Consultado en: 1505-01.

http://www.um.es/gtiweb/portales/PORTALES-construccion.pdf

25. Fitzloff, Emily, op. cit.

26. Maldonado, Javier. “¿Cómo planificar una campaña de banners?”. En: Pc-actual, 2000, abril. Consultado en: 16-08-00.

http://www.marketalia.com/articulos/000002.html

27. Sanmartín, N. "La venta de guerrilla se adueña de internet". En: Cinco días, 2000. Consultado en: 31-07-00.

http://www.cincodias.es/scripts/cincodias/noticias/articulo.asp? ntc $=1553$ $27 \& a p=2$

http://www.digiteldocs.com/PIE-Sectorial-Files/1327.html 